\title{
Designing, Optimization and Validation of Tetra Primer ARMS-PCR Protocol for Genotyping Single Nucleotide Polymorphism rs4731702 (C/T) of KLF14 Gene Associated with Type 2 Diabetes Mellitus: A Study in San Luis, Argentina
}

\author{
Micaela Fernanda Alvarez, Miriam Ester Vasquez Gomez, Susana Siewert \\ Laboratory of Diabetes, Department of Biochemistry and Biological Sciences, School of Chemistry, Biochemistry and \\ Pharmacy, National University of San Luis, San Luis, Argentina \\ Email: ssiewert@unsl.edu.ar
}

How to cite this paper: Alvarez, M.F., Gomez, M.E.V. and Siewert, S. (2016) Designing, Optimization and Validation of Tetra Primer ARMS-PCR Protocol for Genotyping Single Nucleotide Polymorphism rs4731702 (C/T) of KLF14 Gene Associated with Type 2 Diabetes Mellitus: A Study in San Luis, Argentina. Open Access Library Journal, 3: e3270.

http://dx.doi.org/10.4236/oalib.1103270

Received: December 1, 2016

Accepted: December 12, 2016

Published: December 16, 2016

Copyright $\odot 2016$ by authors and Open Access Library Inc.

This work is licensed under the Creative Commons Attribution International

License (CC BY 4.0).

http://creativecommons.org/licenses/by/4.0/

\begin{abstract}
New, quick, and inexpensive methods for genotyping KLF14 gene polymorphism through Tetra Primer ARMS-PCR were developed in the present investigation. Single nucleotide polymorphism (SNP) genotyping needs to be attempted to establish association between the identified SNP with metabolic trait for identification of molecular markers that can be used to identify individuals at risk of developing Diabetes Mellitus Type 2. In the current study, we have successfully genotyped the SNP rs4731702 (C/T) in KLF14 gene. Tetra primer ARMS-PCR protocol was optimized and validated for this SNP with short turn-around time and costs. The optimized techniques were tested on 60 samples of controls and type 2 Diabetes Mellitus individuals. Samples with known genotypes for the described gene, previously tested in duplicate using the sequencing methods, were employed for validation of the assay. Upon validation, complete concordance was observed between the Tetra Primer ARMS-PCR assays and the sequencing results. These results highlight the ability of Tetra Primer ARMS-PCR in genotyping of SNP in KLF14 gene. Our results provide direct evidence that Tetra Primer ARMS-PCR is a rapid, reliable, and cost-effective method for SNP genotyping of KLF14 gene in type 2 Diabetes Mellitus individuals.
\end{abstract}

\section{Subject Areas}

Genetics

\section{Keywords}

Molecular Markers, SNP, Insulinemia, Metabolic Syndrome, Dyslipidemias 


\section{Introduction}

Type 2 Diabetes Mellitus (T2DM) is a chronic disease also called adult-onset diabetes or non-insulin-dependent diabetes and accounts for more than $90 \%$ of all diabetes types in the global population. Without proper monitoring, it can lead to serious complications such as cardiovascular disease, kidney disease, nerve disease, and eye disease. T2DM is a life-long condition that requires careful management. The incidence and prevalence have reached epidemic proportions worldwide [1].

The physiological disturbances of T2DM include abdominal obesity, insulin resistance, elevated low density lipoprotein cholesterol (LDL-c), elevated triglycerides (TG), reduced high density lipoproteins cholesterol (HDL-c) and hypertension. Although environmental factors, particularly caloric excess and physical inactivity, play a role in T2DM; the traits are highly heritable.

To date, approximately 25 genome-wide significant common variant associations with T2DM have been described, mostly through genome-wide association analyses (GWAS) [2]-[12]. The identities of the variants and genes mediating the susceptibility effects at most of these signals have yet to be established, and the known variants account for less than $10 \%$ of the overall estimated genetic contribution to T2DM predisposition.

Although GWAS have identified numerous loci influencing these traits individually, but to date, no loci have been found that affect the entire spectrum of T2DM traits. Variants near $K L F 14$, which encodes the transcription factor Krüppel-like Factor 14, have previously been associated with T2DM and HDL cholesterol levels in large GWAS analyses [13]-[18].

The $K L F 14$ is shown to act as a master role in regulating the expression of adipose genes that are associated with key metabolic traits. Interestingly, expression levels of ten genes (TPMT, ARSD, SLC7A10, C8orf82, APH1B, PRMT2, NIN2, KLF13, GNB1, $M Y L 5)$ were associated with a variety of metabolic syndrome traits, including obesity, dyslipidemias and measures of insulin resistance, supporting a trans-causal link between KLF14 expression and these ten genes [19].

The single nucleotide polymorphism (SNP) of rs4731702 14 kb upstream of KLF14 has implicated a high correlation with HDL-c and cardiovascular disease [13] [14] [15] [20].

Maternally derived alleles at rs4731702 in KLF14 are associated with expression of a number of genes that are correlated with insulinemia and other characteristics of metabolic syndrome [19]. Hanson et al. [21] found an association between the diabetes risk allele (C) of the KLF14 SNP rs4731702 and lower insulin sensitivity. The diabetes risk allele also was associated with hyperinsulinemia in a previous study, which suggests increased insulin resistance [16].

Previous functional studies found that the maternally transmitted $\mathrm{T}$ allele of rs4731702 SNP is associated with increased expression of KLF14 in adipose tissue, indicating the presence of a cis expression quantitative trait loci (eQTL) [9]. In addition, the $\mathrm{T}$ allele showed a consistent association with increased HDL-c [20]. 
Therefore this polymorphism need to be associated with metabolic trait for identification of molecular markers that can be used to identify individuals at risk of developing Diabetes Mellitus at an early stage of life using robust and less costly genotyping techniques. A plethora of methods for genotyping of specific polymorphic loci are currently used and these include Polymerase Chain Reaction-Restriction Fragment Length Polymorphism (PCR-RFLP), Single Strand Conformation Polymorphism (SSCP), direct DNA sequencing, and Tetra-Primer Amplification Refractory Mutation SystemPolymerase Chain Reaction (Tetra Primer ARMS-PCR). In developing countries it is very difficult and costly to genotype large number of individuals by methods involving PCR and post-PCR manipulations. To circumvent these problems, simple, fast and cost effective genotyping methods need to be developed. In Tetra Primer ARMS-PCR, 2 pairs of primers in a single PCR tube, can simultaneously amplify both alleles as well as allow amplification of an internal DNA control. This technique has been applied to study different mutations [22] [23] [24]. Ye et al. [25] were the first to describe tetra-primer PCR in which allele-specific amplification is achieved in a single PCR reaction using two outer primers and two allele-specific inner primers. Ye et al. [26] combined tetra-primer PCR with ARMS to form the Tetra Primer ARMS-PCR or T-ARMS technique by introducing deliberate mismatches at position -2 from the 3 ' end of inner primers to improve allele specificity. In a single step reaction, the outer primers amplify a large fragment of the target gene, irrespective of its genotype although each inner primer combines with a particular opposite outer primer to generate smaller allelespecific amplicons, which are of different sizes and can easily be discriminated on gel electrophoresis either as homozygous or heterozygous.

In the present study, we developed rapid, efficient, cost effective and allele specific Tetra Primer ARMS-PCR for genotyping rs4731702 (C/T) polymorphism of KLF14, so that these methods can be used to genotype large samples of individuals in order to estimate association of this SNP with individuals at risk of developing Diabetes Mellitus Type 2.

\section{Research Design and Methods}

\subsection{Subjects}

The present study was carried out in accordance with the guidelines of the Helsinki Declaration. A total of 60 volunteers (30 patients with type 2 diabetes and 30 healthy age-matched controls) participated in this investigation.

Criteria published by the American Diabetes Association were used to diagnose T2DM [27]. These patients reside in San Luis, Argentina. The protocol for this study was approved by the local Institutional Review Board, and a written informed consent was obtained from each patient to be enrolled. During an initial interview with each patient, they were asked for diseases, medication and smoking histories. Exclusion criteria included liver, kidney and thyroid diseases, as well as the use of anti-lipemic drugs.

\subsection{DNA Samples}

Genomic DNA was isolated from diabetic patients and healthy volunteers using con- 
ventional protocol by Qiagen kits (Qiagen, Inc., Valencia, CA). DNA concentration was detected by UV-VIS spectroscopy and diluted to a final concentration of $20 \mathrm{ng} / \mu \mathrm{L}$.

\subsection{Primer Design, Amplification, and Genotyping of Tetra Primer ARMS-PCR}

Based on the GenBank sequence of human KLF14, accession number: NT_007933.15, we designed "in silico" a pair of outer primers and two allele-specific inner primers for Tetra Primer ARMS-PCR, in a free access web (http://cedar.genetics.soton.ac.uk) and then checked for specificity (http://blast.ncbi.nlm.nih.gov/Blast.cgi) (Table 1).

Each PCR reaction was carried out in a total volume of $35 \mu \mathrm{L}$, containing $200 \mathrm{ng}$ of template DNA, 1 pmol of inner primer $\mathrm{C}$ allele, $10 \mathrm{pmol}$ of inner primer allele $\mathrm{T}$ and 10 pmol of each outer primer, $200 \mu \mathrm{M}$ dNTPs, $2.5 \mathrm{mM} \mathrm{MgCl}_{2}, 1 \times$ buffer, and 1 unit of AmpliTaq Gold DNA polymerase (Perkin-Elmer). The template DNA was denatured for 3 minutes at $95^{\circ} \mathrm{C}$ before undergoing 35 cycles of denaturation for 1 minute at $95^{\circ} \mathrm{C}$, primer annealing for 1 minute at $62^{\circ} \mathrm{C}$ and extension for 1 minute at $72^{\circ} \mathrm{C}$, and final extension at $72^{\circ} \mathrm{C}$ for 3 minutes (Table 1). The resultant products obtained after PCR were separated by electrophoresis on $2.5 \%$ agarose gel containing GelRed. The image was visualized and photographed under UV transillumination. Randomly selected $20 \%$ of samples were re-genotyped for cross validating initial genotypes. In case of unclear genotyping results, the samples were repeated again in duplicates till clear genotype was available. No genotyping error was observed during cross validation.

\subsection{Validation of the Assay}

To evaluate the efficiency and accuracy of the assay, selected PCR-amplified DNA samples ( $n=3$, respectively, for each genotype) were examined by DNA sequencing and the results obtained by Tetra Primer ARMS-PCR were compared with those determined by sequencing.

\section{Results}

The SNP [rs4731702 (C/T)], an important candidate for identify individuals at risk of

Table 1. PCR primers and conditions.

\begin{tabular}{|c|c|c|c|}
\hline $\begin{array}{c}\text { Genetic } \\
\text { polymorphism }\end{array}$ & Primer sequence & $\begin{array}{l}\text { Annealing } \\
\text { temperature }\end{array}$ & Amplicon size \\
\hline \multirow{4}{*}{$\begin{array}{l}\mathrm{rs} 4731702 \\
\quad(\mathrm{C} / \mathrm{T})\end{array}$} & $\begin{array}{l}\text { Forward inner primer (C allele): } \\
\text { 5'AAAAAACAGCATTATTTCCCACACAAAC 3' }\end{array}$ & & 515 bp (C allele) \\
\hline & $\begin{array}{c}\text { Reverse inner primer (T allele): } \\
\text { 5' TATCTTTTTGGTGCTAAATGGAACGGA 3' }\end{array}$ & $62^{\circ} \mathrm{C}$ & $58 \mathrm{bp}$ (T allele) \\
\hline & $\begin{array}{c}\text { Forward outer: } \\
\text { 5' CCCAAGGCATCTATCCAAAA 3' }\end{array}$ & & $\begin{array}{c}619 \mathrm{bp} \\
\text { (from two outer } \\
\text { primers) }\end{array}$ \\
\hline & $\begin{array}{c}\text { Reverse Outer: } \\
\text { 5' CCGTTGAACTGTGTTTGCAC 3' }\end{array}$ & & \\
\hline
\end{tabular}


developing Diabetes Mellitus Type 2, was genotyped by Tetra Primer ARMS-PCR based methodology. PCR fragments were generated as per expectations for all the loci. All the PCR products were well resolved and sized by agarose gel electrophoresis, allowing easy identification of different genotypes. Heterozygotes and homozygotes were unambiguously assigned from the gel profile.

The size of DNA fragments amplified with these four primers for rs4731702 (C/T) of KLF14 (619 bp control fragment, 158 bp T allele, 515 bp C allele) was suitable for separation on $2.5 \%$ agarose gels (Figure 1 ).

Several factors, including primer concentration and PCR cycling conditions, which can affect PCR specificity and efficiency were optimized. For sets of outer-inner primer pairs we tested $\mathrm{Mg}^{2+}$ concentrations from $1.5 \mathrm{mM}$ to $4 \mathrm{mM}$ and best results were obtained at $\mathrm{Mg}^{2+}$ concentration of $2.5 \mathrm{mM}$ for the primer sets. Gradient PCR was carried out on the BIORAD iCycler to determine the best annealing temperature for primer sets (change per reaction was $1^{\circ} \mathrm{C}$ ) and optimum annealing temperatures were finalized to be $62^{\circ} \mathrm{C}$ for $\mathrm{rs} 4731702(\mathrm{C} / \mathrm{T})$ loci.

In our study, optimization of primer concentrations was the trickiest one, since the usually suggested outer-inner ratio (1:10) for tetra-primer ARMS PCR was not useful for our primer sets. Thus, we performed PCR with three more ratios $(1: 10,1: 5,1: 1)$ of outer-inner primers and 1:1 was found to give best results for $\mathrm{T}$ allele and 1:10 for the $\mathrm{C}$ allele.

Validation of the developed methodology was done by direct sequencing of representative samples from each set using the outer primers for each SNP. We observed complete concordance between the methods. The genotypes scored from the assay were in $100 \%$ accordance with direct sequencing (Figure 2).

\section{Discussion}

Kruppel-like factors (KLFs) comprise a family of evolutionarily conserved zinc fingercontaining transcription factors with diverse regulatory functions in cell growth, proliferation, differentiation and embryogenesis [28] [29]. In fact, KLF14 has been recently

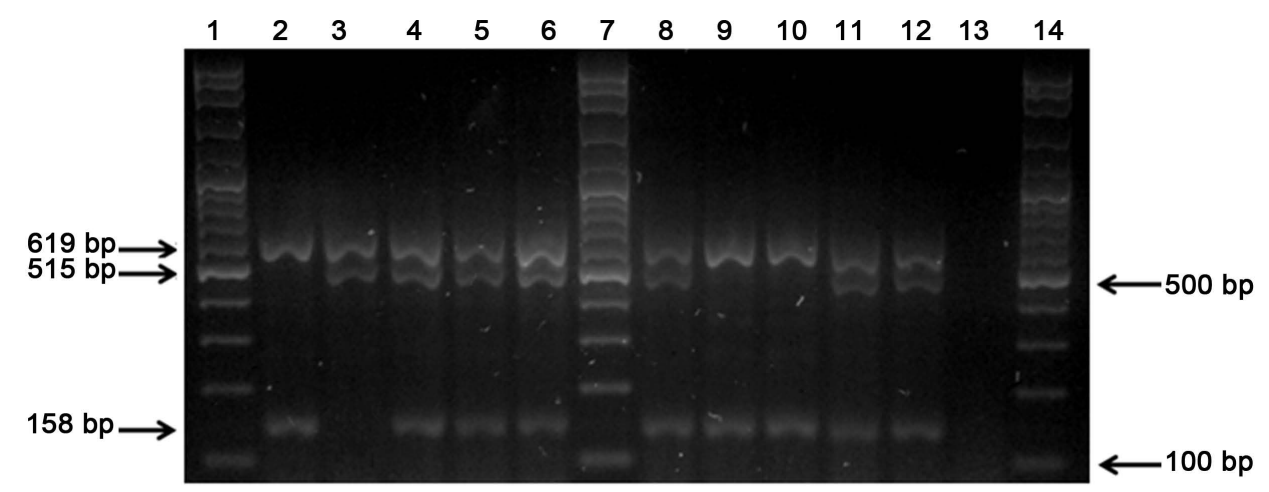

Figure 1. Agarose gel electrophoresis (2.5\%) of polymerase chain reaction (PCR) product of Tetra-primer ARMS-PCR. Lanes 2, 9 and 10 show a typical T/T genotype; lanes 4, 5, 6, 8, 11 and 12 show a typical C/T genotype; lane 3 shows a typical C/C genotype; lane 13 shows (-) control and lanes 1,7 and 14 show the molecular weight marker. 


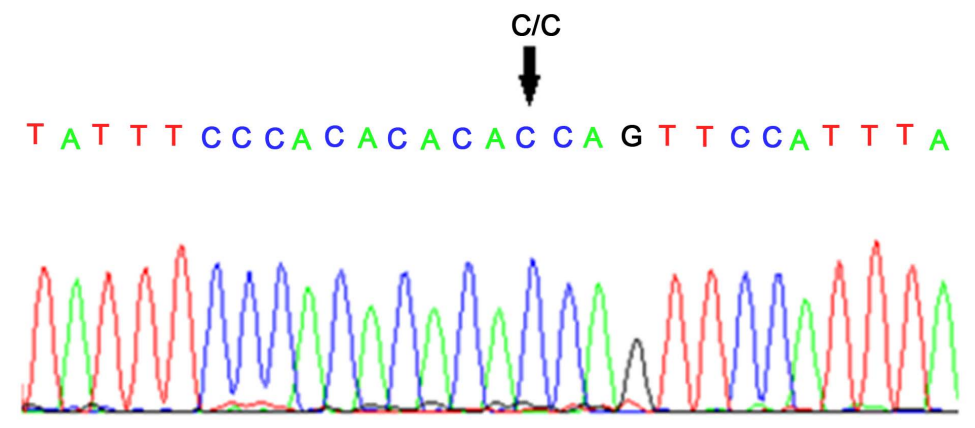

(a)

$\mathrm{T} / \mathrm{T}$

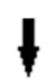

TAT T T C C CACACACA T CA G T T C C A T T T A

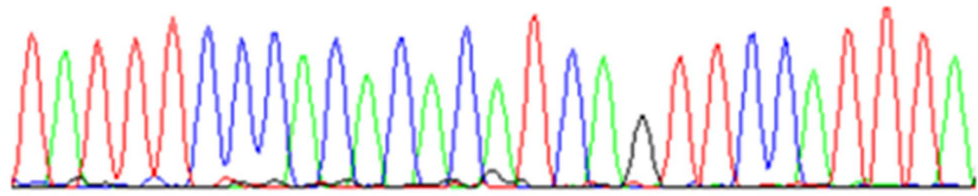

(b)

$\mathrm{C} / \mathrm{T}$
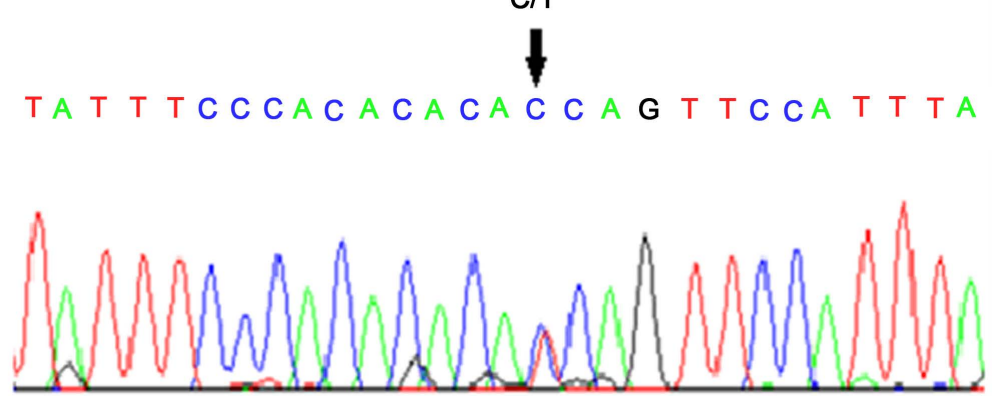

(c)

Figure 2. The sequencing results of three genotypes of the $K L F 14$ gene.

proposed as a master trans-regulator of multiple genes that are associated with metabolic phenotypes in adipose tissue [19], T regulatory cell differentiation [30], and lipid-mediated signaling through a distinct epigenetic mechanism [31].

$K L F 14$ has recently elicited significant attention since extensive genetic studies in humans identified a central role of this protein in the development of metabolic diseases, in particular those that regulate lipid metabolism. In fact, because of its contribution to metabolic diseases, $K L F 14$ has been recently referred to as a "conductor of the metabolic syndrome orchestra" [32].

$K L F 14$, a maternally expressed imprinted gene without introns, is robustly associated with HDL-c levels, cardiovascular disease, Type 2 Diabetes Mellitus, obesity, and cancer [13] [14] [16] [18] [19] [20] [31] [33] [34] [35] [36].

Small et al. [19] demonstrated that the type 2 diabetes and HDL-c associated cis- 
acting eQTL of $K L F 14$ acts as a master trans-regulator of adipose gene expression. The results suggested a trans-causal link between $K L F 14$ expression and ten genes that were associated with a variety of metabolic syndrome traits including obesity, dyslipidemia, and measures of insulin resistance. Moreover, using large scale genome-wide association study data, they showed that five of the ten genes had nearby SNPs that were associated with key metabolic syndrome traits at genome wide significance.

Variants near the transcription factor KLF14 (Kruppel-like factor 14) are robustly associated with both Type 2 Diabetes and HDL-c in large-scale genome-wide association studies conducted in Caucasians [13] [17] [20] [34]. These studies have implicated a group of highly-correlated SNPs including rs4731702 14 kb upstream of KLF14 [13] [17].

Since transcription factors such as KLF14 typically modulate expression of other genes in trans, Small et al. [19] tested for association between rs4731702 and expression levels of $\sim 24 \mathrm{~K}$ probes (16,663 genes) and they focused on ten genes (TPMT, ARSD, SLC7A10, C8orf82, APH1B, PRMT2, NIN/2, KLF13, GNB1, MYL5) showing genomewide significant trans (GWST) associations $\left(p<5 \times 10^{-8}\right)$ driven by rs4731702. Therefore, de Assuncao et al. [31] reported the description of the activity and mechanisms underlying the function of $K L F 14$ as an activator protein and novel regulator of lipid signaling.

In addition, the $\mathrm{T}$ allele of $\mathrm{rs} 4731702$, is significantly associated with HDL-c levels and a decreased risk of cardiovascular disease and type 2 diabetes [10] [13] [34].

Functional studies demonstrate that the maternally transmitted $\mathrm{T}$ allele of rs4731702 SNP is associated with increased expression of $K L F 14$ in adipose tissue, indicating the presence of a cis expression quantitative trait loci (eQTL) [10]. All this evidence indicates that the KLF14 variant is an important regulator of lipoprotein metabolism. In addition, the $\mathrm{T}$ allele carriers have higher ApoA-I levels in the Mulao population in China [33].

Taken together, the rs4731702 SNP may act in cis to influence the KLF14-associated trans-regulatory network and bring about the cascade of events in lipid metabolism.

Chen et al. [20] detected positive associations of KLF14-rs4731702 with atherosclerotic cardiovascular disease, including ischemic stroke and myocardial infarction phenotypes. Thus, the KLF14 gene is not only an important factor for the regulation of metabolic phenotypes, but also involved in the pathogenesis of atherosclerotic-related phenotypes such as ischemic stroke and myocardial infarction.

Hence, for genetic analysis, fast and economical assays that can be performed with standard PCR instruments are highly desirable. Single nucleotide polymorphism (SNP), a novel molecular marker technology, refers to a sequence polymorphism caused by a single nucleotide mutation at a specific locus in the DNA sequence. This sort of polymorphism includes single base transitions, transversions, insertions and deletions [37], and the minor allele frequency should be $1 \%$ or greater. Of all the SNP mutation types, transitions are the most common (approx. 66.6\%) [38]. The workers in the past have used techniques like Polymerase Chain Reaction-Restriction Fragment Length Poly- 
morphism (PCR-RFLP), Single Strand Conformation Polymorphism (SSCP), direct DNA sequencing, and tetra-primer Amplification Refractory Mutation System-Polymerase Chain Reaction (ARMS-PCR) for genotyping of specific polymorphic nucleotide loci. Among these the tetra-primer ARMS-PCR could be a useful tool for genotyping, since SSCP may not be repeatable some times, direct DNA sequencing is a cumbersome, time consuming, technically demanding and costly procedure and the possibility of getting a restriction site for an enzyme could be rare for genotyping by RFLP.

Compared to other genotyping techniques, Tetra Primer ARMS-PCR has been reported to be a rapid, reliable, simple and economical assay for SNP genotyping [26] [39] [40]. In the other hand, Etlik et al. [41] compared tetra-primer ARMS assay with routinely used methods such as typical PCR-RFLP analysis, real time PCR assay and DNA sequencing. They concluded that although real time PCR and DNA sequencing are sensitive and accurate techniques, Tetra Primer ARMS-PCR assay could be beneficial in terms of total time, cost and applicability in a typical laboratory.

Hence Tetra Primer ARMS-PCR based methodology was developed for genotyping rs4731702 $(\mathrm{C} / \mathrm{T})$ in the present study. The assay described here is more convenient than the traditional PCR-RFLP since it eliminates the need for incubation with restriction enzymes. This not only avoids any consequent errors and artifacts from such procedures but also reduces the amount of DNA required for the digestion step in PCRRFLP. No special equipment and only a small amount of standard PCR reagents are needed in Tetra Primer ARMS-PCR.

The Tetra Primer ARMS-PCR methods described above are the first reported method allowing one to genotype the SNP rs4731702 (C/T) of KLF14 gene with no postPCR treatment other than electrophoresis. These methods are rapid, simple, reliable, and easy to perform, economical and require minimum level of expertise that can be used for both large-and small-scale genotyping studies.

In summary, the association studies which link genetic variants with lipid levels and susceptibility of atherosclerotic cardiovascular disease risk may also have important significances in pharmacogenetics for guiding personalized treatment of high lipids, especially in our populations which have a high prevalence of T2DM and metabolic syndrome [42].

\section{Conclusions}

With the rapid advances in molecular techniques, various methods for genotyping single-nucleotide polymorphisms (SNPs) are available. Still, the search for easy, robust, and less costly techniques continues. We wished to develop a Tetra Primer Amplification Refractory Mutation System-Polymerase Chain Reaction based technique for genotyping the SNP rs4731702 (C/T) of KLF14 gene so that these methodologies could be used to establish association between the studied polymorphism and metabolic trait for detection of molecular markers that can be used to identify individuals at risk of developing Diabetes Mellitus Type 2.

Importantly, our study emphasizes the importance of genotyping T2DM patients 
according to the rs4731702 (C/T) polymorphism of the KLF14 gene in order to establish efficient prevention policies to reduce the risk of developing T2DM. Finally, genotyping of the rs4731702 (C/T) polymorphism of the KLF14 gene can be of high predictive and interventional value of cardiovascular complications in our, and probably other, populations which have a high prevalence of T2DM.

\section{Acknowledgements}

This research was supported by grants from the Universidad Nacional de San Luis (SES-PROICO 2-2814).

\section{Conflict of Interests}

The authors declare no conflict of interests with respect to the present paper.

\section{References}

[1] Scully, T. (2012) Diabetes in Numbers. Nature, 485, S2-S3. https://doi.org/10.1038/485S2a

[2] Sladek, R., et al. (2007) A Genome-Wide Association Study Identifies Novel Risk Loci for Type 2 Diabetes. Nature, 445, 881-885. https://doi.org/10.1038/nature05616

[3] Zeggini, E., et al. (2007) Replication of Genome-Wide Association Signals in UK Samples Reveals Risk Loci for Type 2 Diabetes. Science, 316, 1336-1341.

https://doi.org/10.1126/science.1142364

[4] Saxena, R., et al. (2007) Genome-Wide Association Analysis Identifies Loci for Type 2 Diabetes and Triglyceride Levels. Science, 316, 1331-1336. https://doi.org/10.1126/science.1142358

[5] Steinthorsdottir, V., et al. (2007) A Variant in CDKAL1 Influences Insulin Response and Risk of Type 2 Diabetes. Nature Genetics, 39, 770-775. https://doi.org/10.1038/ng2043

[6] Zeggini, E., et al. (2008) Meta-Analysis of Genome-Wide Association Data and Large-Scale Replication Identifies Additional Susceptibility Loci for Type 2 Diabetes. Nature Genetics, 40, 638-645. https://doi.org/10.1038/ng.120

[7] Yasuda, K., et al. (2008) Variants in KCNQ1 Are Associated with Susceptibility to Type 2 Diabetes Mellitus. Nature Genetics, 40, 1092-1097. https://doi.org/10.1038/ng.207

[8] Unoki, H., et al. (2008) SNPs in KCNQ1 Are Associated with Susceptibility to Type 2 Diabetes in East Asian and European Populations. Nature Genetics, 40, 1098-1102. https://doi.org/10.1038/ng.208

[9] Rung, J., et al. (2009) Genetic Variant near IRS1 Is Associated with Type 2 Diabetes, Insulin Resistance and Hyperinsulinemia. Nature Genetics, 41, 1110-1115. https://doi.org/10.1038/ng.443

[10] Kong, A., et al. (2009) Parental Origin of Sequence Variants Associated with Complex Diseases. Nature, 462, 868-874. https://doi.org/10.1038/nature08625

[11] Dupuis, J., et al. (2010) New Genetic Loci Implicated in Fasting Glucose Homeostasis and Their Impact on Type 2 Diabetes Risk. Nature Genetics, 42, 105-116. https://doi.org/10.1038/ng.520

[12] Saxena, R., et al. (2010) Genetic Variation in GIPR Influences the Glucose and Insulin Responses to an Oral Glucose Challenge. Nature Genetics, 42, 142-148.

https://doi.org/10.1038/ng.521 
[13] Teslovich, T.M., et al. (2010) Biological, Clinical and Population Relevance of 95 Loci for Blood Lipids. Nature, 466, 707-713. https://doi.org/10.1038/nature09270

[14] Chasman, D.I., et al. (2009) Forty-Three Loci Associated with Plasma Lipoprotein Size, Concentration, and Cholesterol Content in Genome-Wide Analysis. PLoS Genetics, 5, e1000730. https://doi.org/10.1371/journal.pgen.1000730

[15] Jeemon, P., et al. (2011) Implications of Discoveries from Genome-Wide Association Studies in Current Cardiovascular Practice. World Journal of Cardiology, 3, 230-247. https://doi.org/10.4330/wjc.v3.i7.230

[16] Ohshige, T., et al. (2011) Association of New Loci Identified in European Genome-Wide Association Studies with Susceptibility to Type 2 Diabetes in the Japanese. PLoS ONE, 6, e26911. https://doi.org/10.1371/journal.pone.0026911

[17] Voight, B.F., et al. (2010) Twelve Type 2 Diabetes Susceptibility Loci Identified through Large-Scale Association Analysis. Nature Genetics, 42, 579-589.

https://doi.org/10.1038/ng.609

[18] Rees, S.D., et al. (2011) Replication of 13 Genome-Wide Associations (GWA)-Validated Risk Variants for Type 2 Diabetes in Pakistani Populations. Diabetologia, 54, 1368-1374. https://doi.org/10.1007/s00125-011-2063-2

[19] Small, K.S., et al. (2011) Identification of an imprinted Master Trans Regulator at the KLF14 Locus Related to Multiple Metabolic Phenotypes. Nature Genetics, 43, 561-564. https://doi.org/10.1038/ng.833

[20] Chen, X., et al. (2012) Genome-Wide Association Study Validation Identifies Novel Loci for Atherosclerotic Cardiovascular Disease. Journal of Thrombosis and Haemostasis, 10, 1508-1514. https://doi.org/10.1111/j.1538-7836.2012.04815.x

[21] Hanson, R.L., et al. (2013) Strong Parent-of-Origin Effects in the Association of KCNQ1 Variants with Type 2 Diabetes in American Indians. Diabetes, 62, 2984-2991. https://doi.org/10.2337/db12-1767

[22] Old, J.M., Varawalla, N.Y. and Weatherall, D.J. (1990) Rapid Detection and Prenatal Diagnosis of Beta-Thalassaemia: Studies in Indian and Cypriot Populations in the UK. Lancet, 336, 834-837. https://doi.org/10.1016/0140-6736(90)92338-I

[23] Vannucchi, A.M., Pancrazzi, A, Bogani, C, Antonioli, E and Guglielmelli, P. (2006) A Quantitative Assay for $J A K 2^{\mathrm{V} 617 \mathrm{~F}}$ Mutation in Myeloproliferative Disorders by ARMS-PCR and Capillary Electrophoresis. Leukemia, 20, 1055-1060.

https://doi.org/10.1038/sj.leu.2404209

[24] Wang, Y.Z., et al. (2014) Detection of Hepatitis B Virus A1762T/G1764A Mutant by Amplification Refractory Mutation System. Brazilian Journal of Infectious Diseases, 18, 261-265. https://doi.org/10.1016/j.bjid.2013.09.005

[25] Ye, S., Humphries, S. and Green, F. (1992) Allele Specific Amplification by Tetra-Primer PCR. Nucleic Acids Research, 20, 1152. https://doi.org/10.1093/nar/20.5.1152

[26] Ye, S., Dhillon, S., Ke, X., Collins, A.R. and Day, I.N.M. (2001) An Efficient Procedure for Genotyping Single Nucleotide Polymorphisms. Nucleic Acids Research, 29, E88. https://doi.org/10.1093/nar/29.17.e88

[27] Report of the Expert Committee on the Diagnosis and Classification of Diabetes Mellitus (1997) Diabetes Care, 20, 1183-1197.

[28] Black, A.R., Black, J.D. and Azizkhan-Clifford, J. (2001) Sp1 and Kruppel-Like Factor Family of Transcription Factors in Cell Growth Regulation and Cancer. Journal of Cellular Physiology, 188, 143-160. https://doi.org/10.1002/jcp.1111 
[29] Kaczynski, J., Cook, T. and Urrutia, R. (2003) Sp1- and Kruppel-Like Transcription Factors. Genome Biology, 4, 206. https://doi.org/10.1186/gb-2003-4-2-206

[30] Sarmento, O.F., et al. (2015) A Novel Role for KLF14 in T Regulatory Cell Differentiation. Cellular and Molecular Gastroenterology and Hepatology, 1, 188-202.e4. https://doi.org/10.1016/j.jcmgh.2014.12.007

[31] De Assuncao, T.M., et al. (2014) New Role for Kruppel-Like Factor 14 as a Transcriptional Activator Involved in the Generation of Signaling Lipids. Journal of Biological Chemistry, 289, 15798-15809. https://doi.org/10.1074/jbc.M113.544346

[32] Civelek, M. and Lusis, A.J. (2011) Conducting the Metabolic Syndrome Orchestra. Nature Genetics, 43, 506-508. https://doi.org/10.1038/ng.842

[33] Huang, P., et al. (2013) Association of the KLF14 rs4731702 SNP and Serum Lipid Levels in the Guangxi Mulao and Han Populations. BioMed Research International, 2013, Article ID: 231515. https://doi.org/10.1155/2013/231515

[34] Chen, G., et al. (2012) Genome-Wide Association Study Identifies Novel Loci Association with Fasting Insulin and Insulin Resistance in African Americans. Human Molecular Genetics, 21, 4530-4536. https://doi.org/10.1093/hmg/dds282

[35] Stacey, S.N., et al. (2009) New Common Variants Affecting Susceptibility to Basal Cell Carcinoma. Nature Genetics, 41, 909-914. https://doi.org/10.1038/ng.412

[36] Steegenga, W.T., et al. (2014) Genome-Wide Age-Related Changes in DNA Methylation and Gene Expression in Human PBMCs. Age, 36, 9648.

https://doi.org/10.1007/s11357-014-9648-x

[37] Lander, E.S. (1996) The New Genomics: Global Views of Biology. Science, 274, 536-539. https://doi.org/10.1126/science.274.5287.536

[38] Zhao, Z. and Boerwinkle, E. (2002) Neighboring-Nucleotide Effects on Single Nucleotide Polymorphisms: A Study of 2.6 Million Polymorphisms across the Human Genome. Genome Research, 12, 1679-1686. https://doi.org/10.1101/gr.287302

[39] Little, S. (2001) Amplification-Refractory Mutation System (ARMS) Analysis of Point Mutations. Current Protocols in Human Genetics, Online.

[40] Okayama, N., et al. (2004) Evaluation of a New Efficient Procedure for Single-Nucleotide Polymorphism Genotyping: Tetra-Primer Amplification Refractory Mutation System-Polymerase Chain Reaction. Clinical Chemistry and Laboratory Medicine, 42, 13-16. https://doi.org/10.1515/CCLM.2004.004

[41] Etlik, O., Koksal, V., Arican-Baris, T.S. and Baris, I. (2008) An Improved Tetra-Primer PCR Approach for the Detection of the FGFR3 G380R Mutation Responsible for Achondroplasia. Molecular and Cellular Probes, 22, 71-75. https://doi.org/10.1016/j.mcp.2007.06.002

[42] Siewert, S., Filipuzzi, S., Codazzi, L., Gonzalez, I. and Ojeda, M. (2007) Impact of Metabolic Syndrome Risk Factors in First-Degree Relatives of Type 2 Diabetic Patients. Review of Diabetic Studies, 4, 177-184. https://doi.org/10.1900/RDS.2007.4.177 
Submit or recommend next manuscript to OALib Journal and we will provide best service for you:

- Publication frequency: Monthly

- 9 subject areas of science, technology and medicine

- Fair and rigorous peer-review system

- Fast publication process

- Article promotion in various social networking sites (LinkedIn, Facebook, Twitter, etc.)

- Maximum dissemination of your research work

Submit Your Paper Online: Click Here to Submit

Or Contact service@oalib.com 